\title{
A Wearable Magnetic Field Based Proximity Sensing System for Monitoring COVID-19 Social Distancing
}

\author{
Sizhen Bian \\ sizhen.bian@dfki.de \\ German Research Center for Artificial Intelligence \\ Kaiserslautern, Germany \\ Hymalai Bello \\ Hymalai.Bello@dfki.de \\ German Research Center for Artificial Intelligence \\ Kaiserslautern, Germany
}

\begin{abstract}
We present a wearable, oscillating magnetic field-based proximity sensing system to monitor social distancing as suggested to prevent COVID 19 spread (being between 1.5 and $2.0 \mathrm{~m}$ ) apart. We evaluate the system both in controlled lab experiments and in a real life large hardware store setting. We demonstrate that, due physical properties of the magnetic field, the system is much more robust than current BT based sensing, in particular being nearly $100 \%$ correct when it comes to distinguishing between distances above and below the $2.0 \mathrm{~m}$ threshold.
\end{abstract}

\section{CCS CONCEPTS}

- Human-centered computing $\rightarrow$ Ubiquitous computing.

\section{KEYWORDS}

Magnetic Field; Proximity Sensing; COVID-19; Social Distancing

\section{ACM Reference Format:}

Sizhen Bian, Bo Zhou, Hymalai Bello, and Paul Lukowicz. 2020. A Wearable Magnetic Field Based Proximity Sensing System for Monitoring COVID-19 Social Distancing. In Proceedings of the 2020 International Symposium on Wearable Computers (ISWC '20), September 12-16, 2020, Virtual Event, Mexico. ACM, New York, NY, USA, 5 pages. https://doi.org/10.1145/3410531.3414313

\section{INTRODUCTION}

Social distancing defined as keeping between 1.5 to 2.5 meter distance, together with contact tracing, is widely recognised as one of the most important contributions to the containment of epidemic spread such as COVID-19. Thus, given a contagious patient, it is imperative to find other people whom he/she might have infected. Here the "magic" 1.5 -2.5 meter distance is again of importance: anyone who has been within this distance of the contagious person for a significant amount of time could potentially have been

Permission to make digital or hard copies of all or part of this work for personal or classroom use is granted without fee provided that copies are not made or distributed for profit or commercial advantage and that copies bear this notice and the full citation on the first page. Copyrights for components of this work owned by others than ACM must be honored. Abstracting with credit is permitted. To copy otherwise, or republish, to post on servers or to redistribute to lists, requires prior specific permission and/or a fee. Request permissions from permissions@acm.org.

ISWC '20, September 12-16, 2020, Virtual Event, Mexico

(c) 2020 Association for Computing Machinery.

ACM ISBN 978-1-4503-8077-5/20/09 . \$ \$15.00

https://doi.org/10.1145/3410531.3414313

\author{
Bo Zhou \\ bo.zhou@dfki.de \\ German Research Center for Artificial Intelligence \\ Kaiserslautern, Germany \\ Paul Lukowicz \\ paul.lukowicz@dfki.de \\ German Research Center for Artificial Intelligence \\ Kaiserslautern, Germany
}

infected. Since recalling (or in many cases even knowing) all the people who have been within such a proximity for the last 14 days (the consensus maximum incubation period) is impossible, there has been significant interest in automatic sensor-based solutions. Today contact tracing apps that use Bluetooth proximity sensing have been deployed world wide. While they have the advantage of being easily deployable as they require nothing more than a standard smartphone, they tend to suffer under the well known accuracy and reliability problems $[5,8,12]$. For everyday consumer applications the ease of deployment of smartphone-based solutions may out-weight the accuracy problems. Yet for other more sensitive settings, such as healthcare, critical industries, or education (opening schools in the midst of a pandemic), there has been significant interest in more reliable dedicated proximity sensing solutions. Ultra-sound [7] can give higher distance accuracy but suffer from the robustness of the signal. Capacitive body sensing [1] enjoys low cost, low power consumption but relies on dynamic environment change to generate meaningful results. Other viable methods including thermal image [13], cellular and GPS[14], AI-based computer version $[3,4,11]$ are often under privacy policy debate, as the users' visual appearance or exact location path reveals more information than that needed for mere contact detection.

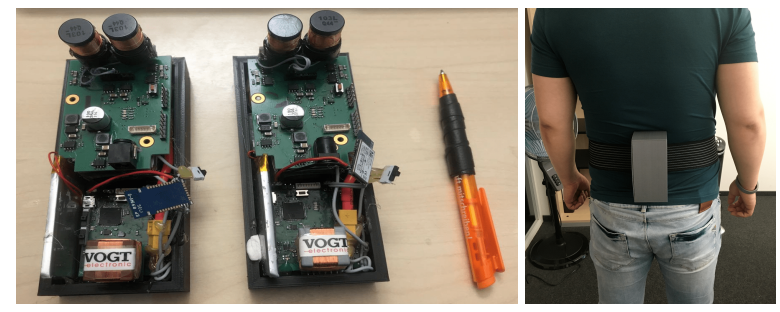

Figure 1: The distancing detection prototype hardware

In this paper, we demonstrate how oscillating magnetic field localisation technology $[9,10]$ can be adapted into compact, wearable, highly accurate proximity tracing system. We leverage the fact that the magnetic field strength is attenuated at cubic relationship with the distance: generating magnetic fields covering indoor scenarios, such as room, hallway, or factories, typically requires strong stationary sources with large coils with high inductance. Smaller oscillating magnetic field systems suitable for wearable 
applications generate just enough radius for the range of social distancing. Different from the concept of localisation, our approach essentially creates a small magnetic field 'bubble' surrounding the moving user for their social distancing zone.

Overall, we have the following contributions:

(1) The general concept for using oscillating magnetic field for contact tracing,

(2) A wearable prototype systems optimised for highly reliable detection of proximity around $2.0 \mathrm{~m}$.

(3) Evaluation of the systems both in the lab and in a real life construction material market setting showing near perfect detection of the required social distance of around $2 \mathrm{~m}$.

\section{APPROACH}

\subsection{General Principle}

The strength of a magnetic field measured at a distance $d$ from its coil (assume the size of the coil is negligible compared to $d$ ) is proportional to $\frac{1}{d^{3}}$. An alternating current through a coil generates an oscillating magnetic field. According to Faraday's law of induction, if another coil is placed in such an oscillating field, voltage proportional to the magnetic field will be induced in that coil. This is the basic physical principle behind our work. The basic principle has two advantages over radio frequency (and to a degree also ultrasound based) approaches:

(1) With coils of a size that is reasonable in a wearable system (2-3cm diameter) and typical currents (not more half ampere) the field is virtually undetectable outside a range of $2-3 \mathrm{~m}$.

(2) The signal is NOT a propagating wave (such as RF or ultrasound signals) but a closed-loop field. Thus, we do not have to deal with refraction, reflection and the resulting multi-path effects.

(3) Magnetic fields are not significantly affected by objects in the environment. However, large ferromagnetic objects do distort the field and is a problem when we want to address in the future.

The systems worn by different users generate their magnetic field impulses by time domain multiplexing (TDM). While in the experiment we used a central server sending Zigbee signals for synchronisation, the protocol can also work in an asynchronous way similar to the ALOHA WiFi protocol. To this end each system (1) sends impulses at random time intervals, (2) when receiving a pulse (other then its own pulse) broadcasts its ID over RF (Zignee or BT).

\subsection{Implementation}

Figure 1 and 2 illustrate two prototypes and the architecture of the system. It consists of transmitter and receiver sub-systems.

The transmitter sub-system generates the magnetic field with a frequency of $20 \mathrm{kHz}$ by driving a $\mathrm{H}$-bridge of two serially connected commercial $3.3 \mathrm{mH}$ inductors. The low resistance of the coil benefits a high resonant voltage after tuning to the resonant circuit. The coil at the receiver side with a value of $10 \mathrm{mH}$ is specially crafted with $\mathrm{MnZn}$-ferrite material, contributing to low losses and high saturation induction. Three sets of coil construct a cube structure to measure the three-dimensional magnetic field.
The receiver sub-system uses tri-axis coils to measure the induced voltage, which is then filtered, amplified (Figure 3) by a 4-order Butter-worth filter and a logarithmic amplifier, which aims to compress the signal with a wide dynamic range to its decibel equivalent via a precise nonlinear transformation. A 24-bits resolution analog-to-digital converter finally samples the voltage with rate of $166,7 \mathrm{~Hz}$.

For evaluation, the data is stored locally into the SD card. The receiver sub-system consumes $120 \mathrm{~mA}$ current at $3.7 \mathrm{~V}$. The transmitter sub-system consumes $170 \mathrm{~mA}$ current at $10 \mathrm{~V}$. A rechargeable $1500 \mathrm{mAH}$ battery supplies the power with an $11.1 \mathrm{~V}$ output and supports a working time of more than five hours. For ease of evaluation, we use Zigbee to synchronise the transmitters, which can be discarded for practical social distancing systems. The size of one system is $16 \mathrm{~cm} \times 6.5 \mathrm{~cm} \times 6 \mathrm{~cm}$. However, the size of our prototype is restricted by the modular boards and the big rechargeable batteries. The system architecture from Figure 2 can be optimized and integrated into smaller footprints in the future.

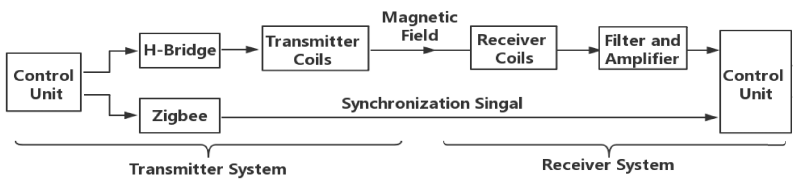

Figure 2: System Architecture

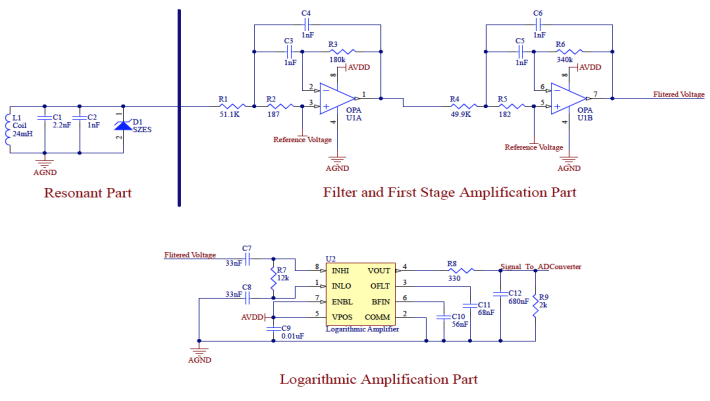

Figure 3: Schematic of the Butterworth filter and logarithmic amplification chain.

\section{LAB EVALUATION}

\subsection{Sensing Range Test}

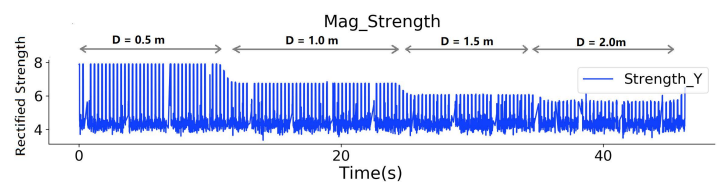

Figure 4: Detection range test with one transmitter activated

To establish a baseline for our implementation, we moved a receiver away from a transmitter in a controlled tabletop experiment. 
Figure 4 shows the received rectified magnetic field strength with different distances between the activated transmitter and the receiver $(0.5$ to $2.0 \mathrm{~m}){ }^{1}$ Since the transmitter only activates the field on one axis and the receiver was not rotated during the experiment, the receiver coil in $\mathrm{Y}$ axis shows the most relevant signal, which will level off beyond $2 \mathrm{~m}$. This means that we either have to mount all devices in the same orientation on the body (which we did for our prototypes) or have a transmitter transmit on all three axis (which is essentially just increases circuit complexity which we wanted to avoid, but have done for our magnetic indoor positioning [10]).

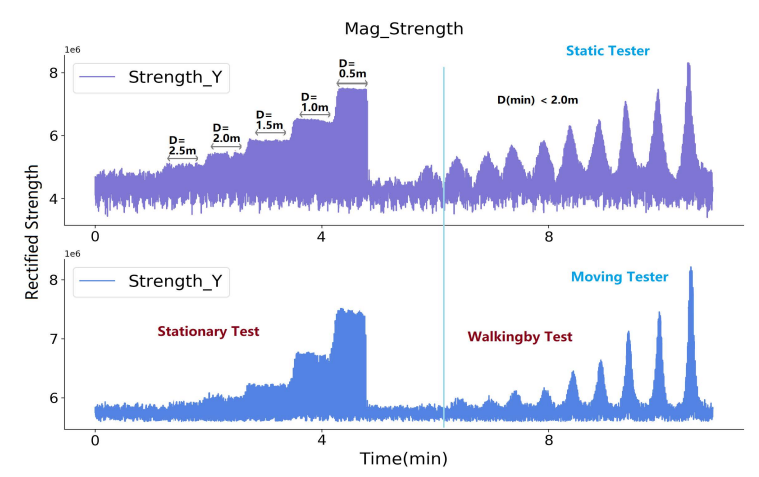

Figure 5: Detection range test with two body-worn systems. One in stationary state and another in mobile state

A second detection range test was performed by deploying the devices on the users. Two volunteers took part in the test. Firstly two testers stood still with different distances from $3 \mathrm{~m}$ to $0.5 \mathrm{~m}$. Then the systems were switched off for another walking-by test, where one tester stood still and another did walking-by events with different minimum distances. Figure 5 shows the sampled strength signal, again we see a detection range beyond $2.0 \mathrm{~m}$. We also noticed that the receivers have different sensitivity which needs to be individually calibrated. Thus to deduce the actual distance information, we used a curve fitting method as exemplified in Figure 6 for each of the prototypes(for tracking social distancing, accuracy with $\mathrm{cm}$ level is not a demand).

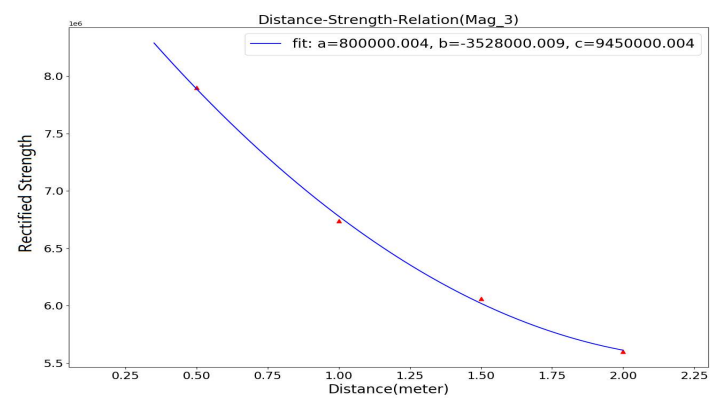

Figure 6: Fitted strength-distance relation of one system

\footnotetext{
${ }^{1}$ The Rectified Strength depicted in all figures is not the physical magnetic field strength with the unit of Tesla. It is the raw value from the analog to digital converter, representing the strength in a way.
}

\subsection{Sensing Robustness Test}

Next we investigated the robustness of our system with respect to disturbance through different objects in the environment. We placed two systems on two tables with a fixed $1.5 \mathrm{~m}$ distance. Different obstacle objects from Figure 7 were placed once at a time between the two devices. The transmitters were deactivated while switching the objects. We tested six everyday objects in our daily life: human body, metal grille, magnetic white board, sofa with wood and metal, thick textile, thick wood. As shown in Figure 8, with period A having nothing between the systems and the other periods with the six everyday objects in between, the registered signal strength was not altered by those obstacles, demonstrating the robustness of our proximity sensing modality.

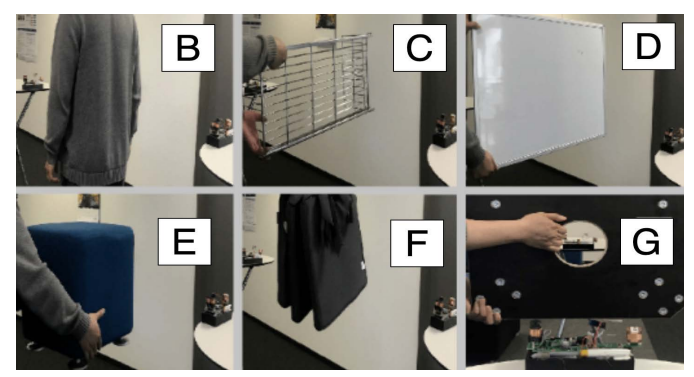

Figure 7: Robustness test with various obstacles between two prototypes

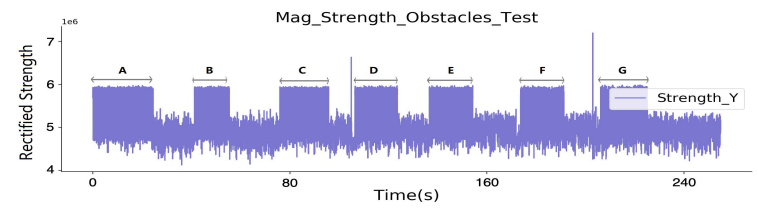

Figure 8: Received strength signals from two prototypes after removing demo's own magnetic field signal

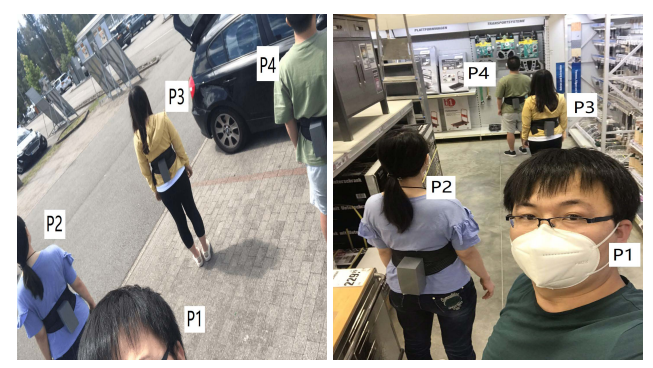

Figure 9: Practical Test outside and inside the construction material supermarket 


\section{REAL LIFE EXPERIMENT}

To demonstrate the feasibility of our approach in a practical scenario, four volunteers worn the prototype systems on the body and walked outside and inside a large hardware store, where a more complex and dynamic environment exists. All volunteers wore the face masks during the test. In addition we ran a simple Bluetooth scanner application where each of our phones broadcast a legible ID (to circumvent the BT MAC anonymizaton strategy [2],) while at the same time scanning for signals from the other phones (based on their known IDs). This was to provide an illustration of how our system compares to established contact tracking $[5,6,8]$ approaches.

As Figure 9 shows, At the beginning of the evaluation the four volunteers stood close to each other outside the supermarket. After that they pared up with one pair keeping a distance of less than $1 \mathrm{~m}$ while the other tried for $1.5 \mathrm{~m}$ (around 2 arms lengths). The distance between groups was beyond $3 \mathrm{~m}$. In that formation the groups walked through the market for around $14 \mathrm{~min}$.
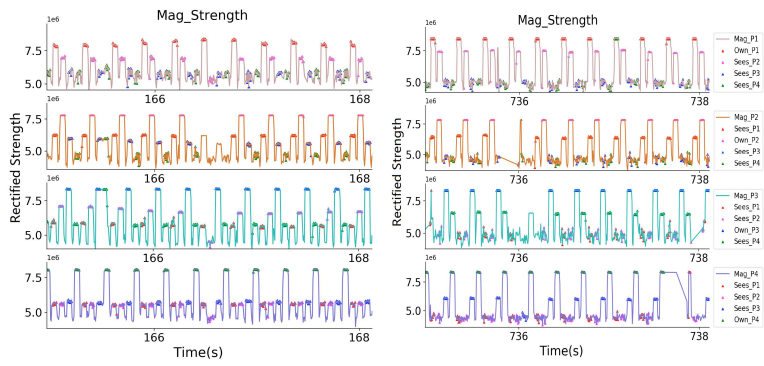

Figure 10: TDM transmission/reception when the four volunteers are below (left) and above (right) the $2-2.5 \mathrm{~m}$ range.

Figure 10 gives a closer look of the sampled raw strength signal with two $3 s$ window showing how the volunteer "sees" the others. Figure 11 gives an overview of the observed magnetic signal strength for each volunteer after the strength data is smoothed by a moving average method with a 10 seconds window, which clearly shows the sensed field strength variation during the whole test. ${ }^{2}$ At the starting and ending period, all the other volunteers could be "seen", and during the middle period, only one volunteer was within the detection range for each volunteer. After interpreting the strength information into the practical distance with a quadratic equation(Figure 6), the social distance of each volunteer is showed in Figure 12. Although we have no ground-truth distancing data during the test, the similarity of "P1_sees_P2" and "P2_sees_P1"(or "P3_sees_P4" and "P4_sees_P3") could demonstrate the truth of distance between them in a way.

In contrast, as Figure 13 shows the Bluetooth RSSI signal supplied by the smartphones. It can be seen that the difference of the signal strength are not nearly as pronounced as in the case of the proposed magnetic field sensor. While for P1 (top diagram) the signal from his immediate partner walking next to him (P2) is consistently stronger then any other signal (as would be expected and desirable), this is neither the case for P3 or P4.

\footnotetext{
${ }^{2}$ The scale of each signal strength in Figure 11 is not identical, which is reasonable since the hardware cannot supply the exact same level of reference voltage in level of $m v$ (Figure 3).
}

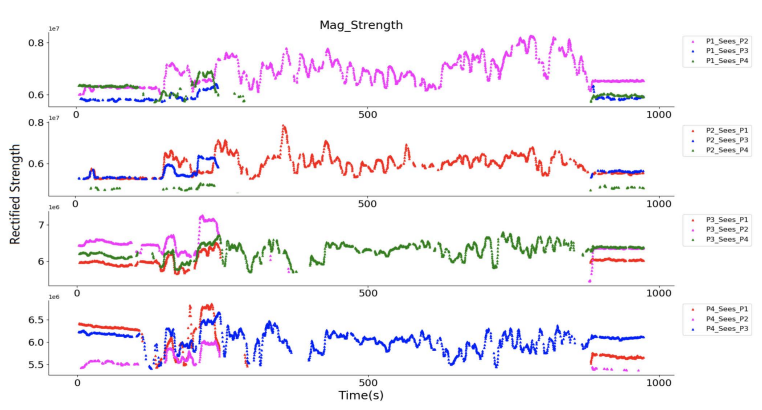

Figure 11: Magnetic field signal strength of each system in the whole test

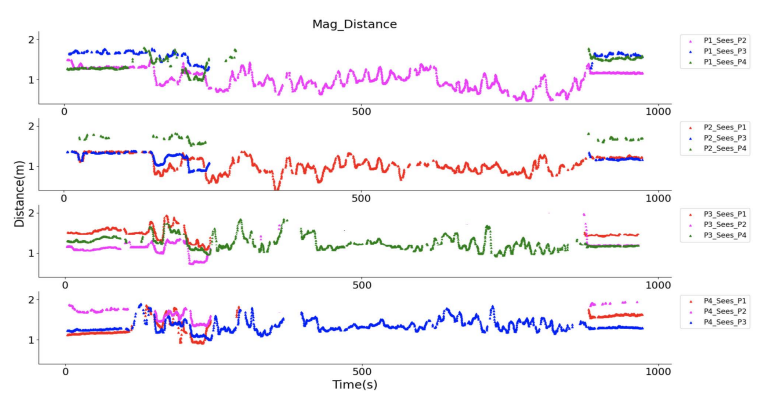

Figure 12: Distance Tracking of Four Volunteers in a construction material supermarket

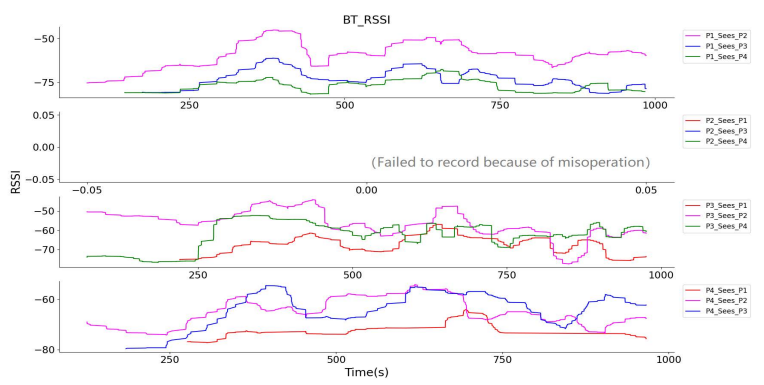

Figure 13: BT-RSSI signal strength of each phone in the whole test

\section{CONCLUSION}

Regarding the COVID-19 caused global level social distancing rule, we designed magnetic field-based proximity sensing prototype systems as a sensing media being able to track a safety social distancing. The wearable system owns a detection range of beyond $2 \mathrm{~m}$ and is robust to everyday environments. A practical evaluation showed that this system could efficiently track the individual's social distancing in real-time and enjoys higher reliability than Bluetooth RSSI signal based social distance tracking system. Future work will be focused on the privacy scheme, firstly using asynchronous protocol for a mass deployment scenario, secondly combining this sensing modality with the Bluetooth MAC anonymization strategy to achieve a privacy well-considered system. 


\section{REFERENCES}

[1] Sizhen Bian, Vitor F Rey, Junaid Younas, and Paul Lukowicz. 2019. Wrist-Worn Capacitive Sensor for Activity and Physical Collaboration Recognition. In 2019 IEEE International Conference on Pervasive Computing and Communications Workshops (PerCom Workshops). IEEE, 261-266.

[2] Guillaume Celosia and Mathieu Cunche. 2019. Saving private addresses: an analysis of privacy issues in the bluetooth-low-energy advertising mechanism. In Proceedings of the 16th EAI International Conference on Mobile and Ubiquitous Systems: Computing, Networking and Services. 444-453.

[3] Marco Cristani, Alessio Del Bue, Vittorio Murino, Francesco Setti, and Alessandro Vinciarelli. 2020. The Visual Social Distancing Problem. arXiv preprint arXiv:2005.04813 (2020)

[4] Abhinav Goel, Aditya Chakraborty, Akhil Chinnakotla, Ashley Kim, Caleb Tung, Damini Rijhwani, Fischer Bordwell, Gore Kao, Isha Ghodgaonkar, Kate Lee, et al. 2020. Using Network Cameras to Observe COVID-19 Social Distancing. (2020).

[5] Hossein Gorji, Markus Arnoldini, David F Jenny, Wolf-Dietrich Hardt, and Patrick Jenny. 2020. STeCC: Smart Testing with Contact Counting Enhances Covid-19 Mitigation by Bluetooth App Based Contact Tracing. medRxiv (2020).

[6] Jinfeng Li and Xinyi Guo. 2020. COVID-19 Contact-tracing Apps: A Survey on the Global Deployment and Challenges. arXiv preprint arXiv:2005.03599 (2020).

[7] Namya Malik. 2020. Social Distancing Sensor: Devices that Use Ultrasound and Radio Frequency Communication to Facilitate Social Distancing. (2020).
[8] Scott McLachlan, Peter Lucas, Kudakwashe Dube, Graham A Hitman, Magda Osman, Evangelia Kyrimi, Martin Neil, and Norman E Fenton. 2020. Bluetooth Smartphone Apps: Are they the most private and effective solution for COVID-19 contact tracing? arXiv preprint arXiv:2005.06621 (2020).

[9] Valter Pasku, Alessio De Angelis, Guido De Angelis, Darmindra D Arumugam, Marco Dionigi, Paolo Carbone, Antonio Moschitta, and David S Ricketts. 2017. Magnetic field-based positioning systems. IEEE Communications Surveys \& Tutorials 19, 3 (2017), 2003-2017.

[10] Gerald Pirkl and Paul Lukowicz. 2013. Resonant magnetic coupling indoor localization system. In Proceedings of the 2013 ACM conference on Pervasive and ubiquitous computing adjunct publication. 59-62.

[11] Narinder Singh Punn, Sanjay Kumar Sonbhadra, and Sonali Agarwal. 2020. Monitoring COVID-19 social distancing with person detection and tracking via finetuned YOLO v3 and Deepsort techniques. arXiv preprint arXiv:2005.01385 (2020).

[12] Manish Shukla, Sachin Lodha, Gautam Shroff, Ramesh Raskar, et al. 2020. Privacy Guidelines for Contact Tracing Applications. arXiv preprint arXiv:2004.13328 (2020).

[13] RA States and E Pappas. 2006. Precision and repeatability of the Optotrak 3020 motion measurement system. fournal of medical engineering \& technology 30,1 (2006), 11-16.

[14] Spencer Woody, Mauricio Garcia Tec, Maytal Dahan, Kelly Gaither, Michael Lachmann, Spencer Fox, Lauren Ancel Meyers, and James G Scott. 2020. Projections for first-wave COVID-19 deaths across the US using social-distancing measures derived from mobile phones. medRxiv (2020). 